\title{
Results of Tobramycin Inhalation Therapy in Patients with Noncystic Fibrosis Bronchiectasis with Pseudomonas aeruginosa Colonization: Real Life Management
}

\author{
Elif Tanriverdi, MD, Binnaz Zeynep Yildirim, MD, Sule Gul, MD, Efsun Gonca Ugur Chousein, MD, \\ Demet Turan, MD, Halit Çınarka, MD, Mehmet Akif Özgül, MD, and Erdoğan Cetinkaya, MD
}

\begin{abstract}
Background: Inhaled antibiotics for treating bronchiectasis have been investigated in the cystic fibrosis population since 1981 and long-term clinical benefits have been reported. However, studies on noncystic fibrosis bronchiectasis (NCFB) have only been performed more recently. Owing to limited evidence, inhaled antibiotics are not currently approved for treating NCFB by the U.S. Food and Drug Administration and the European Medicines Agency. The aim of this study was to evaluate the efficacy and safety of tobramycin inhalation therapy in patients with bronchiectasis with Pseudomonas aeruginosa (PA) colonization.

Methods: In this retrospective cross-sectional study, NCFB patients who were Pseudomonas positive on three consecutive cultures 1 month apart and receiving tobramycin inhalation therapy were evaluated. Evaluation of the following parameters was done in this study: age, gender, smoking history, symptoms, pulmonary function test results, sputum culture results, tobramycin treatment duration, side effects of tobramycin and response evaluation, and hospital admissions before and after treatment. Treatment with $300 \mathrm{mg}$ tobramycin through nebulizer twice daily for 28 days on-off cycles for a total of 6 months was considered to be one treatment period. The approvals for the study were received by the local ethics committee and institutional review board.

Results: Of the 27 patients, 21 patients completed the first period, 7 patients completed the second period, 4 patients completed the third period, and 1 patient completed the fourth period. Sputum culture was negative in $10(47.6 \%)$ of the 21 patients who completed the first period. Decreased sputum purulence and quantity, dyspnea, and cough were observed during treatment. The frequency of hospitalizations before treatment was $1.24 \pm 1.36$, whereas after treatment, it decreased to $0.52 \pm 0.91$, this difference was statistically significant $(p=0.019)$. The most common side effect was increased dyspnea after nebulization in five patients.

Conclusion: Tobramycin inhalation appears to be a well-tolerated treatment in patients with PA colonization with bronchiectasis. This treatment may decrease the hospitalization rates and improve the symptoms.
\end{abstract}

Keywords: bronchiectasis, nebulization, Pseudomonas colonization, tobramycin

Introduction

B RONCHIECTASIS IS DEFINED as a structural distortion and local/diffuse permanent bronchial dilatation of the bronchial tree with peribronchial and bronchial destruction resulting from recurrent bacterial infections and inflammation. Bronchiectasis is divided into two groups: cystic fibrosis (CF) and noncystic fibrosis bronchiectasis (NCFB). NCFB may be of congenital origin such as primary ciliary dyskinesia and primary immunodeficiency or acquired due to tuberculosis, foreign body aspiration, pneumonia, bronchial tumors, rheumatoid arthritis, and ulcerative colitis. ${ }^{(1,2)}$ However, in $\sim 50 \%$ of cases, the underlying cause is still unknown. ${ }^{(3)}$ The presence of systemic inflammation, chronic colonization with Pseudomonas aeruginosa (PA), and severe exacerbations are factors associated with the progression of

Department of Pulmonology, Yedikule Pulmonary Diseases and Thoracic Surgery Education and Research Hospital, Istanbul, Turkey. 
bronchiectasis. ${ }^{(4)} \mathrm{PA}$ is responsible for $21.4 \%$ of bacterial colonization in patients with bronchiectasis and is associated with an increasing loss of pulmonary function, poor quality of life, increase in hospitalizations and exacerbations, and increased mortality compared with other bacteria. ${ }^{(5,6)}$

An increased incidence of NCFB was observed in the past decade as a result of greater access to clinical data and diagnostic tests. This increase led to an increase in treatment costs regardless of the underlying reason. The average annual cost of treatment for patients with chronic infectious disease caused due to PA has been estimated to be almost twofold compared with that for noninfected patients. Inhaled antibiotics for treating bronchiectasis have been investigated in the CF population since 1981 and long-term clinical benefits have been reported. However, studies on NCFB were started only in the past 10 years. Owing to the limited study evidence, inhaled antibiotics are not currently approved for treating NCFB by the U.S. Food and Drug Administration and the European Medicines Agency. ${ }^{(7)}$

This study was conducted to evaluate the results of patients with NCFB receiving inhaled tobramycin for PA colonization after obtaining approval from the Turkish Ministry of Health for nonindicative drug use.

\section{Materials and Methods}

The data from 27 patients who received tobramycin treatment for NCFB during 2014-2019 were evaluated retrospectively. Patient data were acquired from the Ministry of Health based on requests for off-label use of inhaled tobramycin. All subjects with NCFB using inhaled tobramycin in our clinic were included. The following parameters were evaluated in this study: age, gender, smoking history, complaints, type/extent/localization of bronchiectasis, results of pulmonary function tests (PFTs), hemogram, C-reactive protein (CRP), and sputum culture results, tobramycin treatment duration, treatment response, drug side effects, and pre-/post-treatment hospital visits.

Bronchiectasis was diagnosed by high-resolution thoracic computed tomography in all patients. Coexistence of chronic obstructive pulmonary disease (COPD) and/or asthma with NCFB was also evaluated. Total IgE and skin prick test results were recorded for those with a diagnosis of asthma according to the hospital records. The use of inhaled corticosteroids was also recorded.

Treatment with intravenous antipseudomonal agents within 3 months before the three sputum cultures was considered as active infection therapy. One month after active infection treatment, patients gave monthly sputum samples for 3 months. Bacterial growth observed in three consecutive sputum samples at least 1 month apart was evaluated as colonization. Inhaled tobramycin was started after three positive culture results despite active infection treatment. Twice-daily treatment with $300 \mathrm{mg}$ of inhalation tobramycin solution was administered through a jet nebulizer in cycles of 28 days with the medication followed by 28 days off for 24 weeks. Treatment with $300 \mathrm{mg}$ tobramycin through nebulizer twice daily for 28 days on-off cycles for a total of 6 months was considered to be one treatment period. The brand of jet nebulizer varied among the patients. CRP levels, liver and kidney function tests, and sputum culture were reevaluated at the end of each period. If sputum culture positivity persisted at the end of 24 weeks, a new period was given for 24 weeks. If the PA was resistant to tobramycin based on sputum culture results, then these isolates were described as resistant to study drug.

We have compared each patients' initial symptoms and PFTs, leukocytes, and CRP values with their last findings and symptoms after the final treatment period.

Hospitalization rates were calculated as frequency per year, starting from 1 year before treatment and ending with the last treatment period. The approvals for the study were received by the local ethics committee and institutional review board.

\section{Statistical analysis}

Data were analyzed using the Statistical Package for Social Sciences (SPSS) version 22.0 for Windows software (IBM SPSS Statistics Data Editor). Descriptive data are represented as the number of participants and frequency. Categorical variables were compared using chi-square and Fisher's exact tests. Continuous variables are represented as mean and standard deviation, and the Shapiro-Wilk test was used to determine whether these variables were normally distributed. The Student's $t$-test and the Mann-Whitney U test were used for comparing continuous variables depending on the normality of their distribution. A $p$-value of $<0.05$ was considered as statistically significant.

\section{Results}

Of the 27 patients, 11 were women and 16 were men. The mean age was $50.5 \pm 3.3$ years. A median number of four lobes (minimum: one, maximum: six) were involved. Thoracic computed tomography revealed varicose bronchiectasis in $23(85 \%)$ and cylindrical bronchiectasis in $4(15 \%)$ patients. Bronchiectasis was bilateral in $21(77.7 \%)$ patients. The most common symptom was sputum observed in $22(81.5 \%)$ patients, and other common symptoms were dyspnea (70.4\%) and cough $(63 \%)$. None of the patients were using clearance devices such as vests or flutters. Patients' demographics and clinical features are summarized in Table 1.

The rate of dyspnea was significantly higher in male patients $(p=0.019)$ (Table 2$)$. There was no history of smoking in five female patients with dyspnea. History of environmental exposure, particularly biomass, was seen in these patients. Of the total 27 patients, 9 (33.3\%) were smoking, all of whom were male patients with dyspnea. The median duration of smoking was 50 pack-years (minimum: 10, maximum: 150). A total of $24(89 \%)$ patients had COPD and $3(11 \%)$ had asthma. The mean forced expiratory volume in 1 second (FEV1)/forced vital capacity (FVC) ratio was $59 \% \pm 14 \%$. All patients were using inhaled corticosteroids. ABPA (allergic bronchopulmonary aspergillosis) was excluded by the pulmonologist and the allergist with skin prick test and total $\mathrm{IgE}$ results in patients with asthma. In total, 14 (52\%) patients had received active infection therapy. In total, 21 patients completed the first period, 7 patients completed the second period, 4 patients completed the third period, and 1 patient completed the fourth period. Two patients did not come for the follow-up. Of the patients who completed treatment, including those requiring second, third, and fourth treatments for successful eradication, 10 of $21(47.6 \%)$ had negative cultures. Of the 10 patients who had eradication, 5 patients remained free of PA in the following year. Five patients had re-emerging PA in the 
Table 1. Characteristics of Patients

\begin{tabular}{lc}
\hline Characteristics & \\
\hline Age (years, mean \pm SD) & $50.5 \pm 3.3$ \\
Gender, $n(\%)$ & $11(40.7)$ \\
Women & $16(59.3)$ \\
Men & \\
Associated diseases, $n(\%)$ & $24(89)$ \\
COPD & $3(11)$ \\
Asthma & $2(7.5)$ \\
Tuberculosis sequela & $1(3.7)$ \\
Measles history in childhood & $1(3.7)$ \\
Rheumatoid arthritis & $1(3.7)$ \\
Kartagener syndrome & $1(3.7)$ \\
Ulcerative colitis & $1(3.7)$ \\
History of lung abscess & \\
Respiratory function tests at beginning of therapy \\
FEV1 \% & $40 \pm 21$ \\
FVC $\%$ & $54 \pm 20$ \\
FEV1/FVC & $59 \pm 14$ \\
Smoking history, $n$ (\%) & \\
Smoker/former smoker & $9(33.3)$ \\
Nonsmoker & $18(66.6)$ \\
Duration of smoking & $2(7.4)$ \\
(pack-years), median & $2(7.4)$ \\
Symptoms, $n$ (\%) & \\
Cough & \\
Sputum & $17(63)$ \\
Dyspnea & $22(81.5)$ \\
Hemoptysis & $19(70.4)$ \\
Wheezing & \\
\hline
\end{tabular}

FEV1, forced expiratory volume in 1 second; FVC, forced volume vital capacity; SD, standard deviation.

following year (median 4 months, minimum: two to maximum: six). Only 1 patient (4.7\%) among the 21 patients developed bacterial resistance, after second period (Fig. 1).

Evaluation of the treatment response showed that $17(77 \%)$ of 22 patients with sputum complaints had decreased sputum purulence and quantity, and $8(42 \%)$ of the 19 patients with dyspnea reported a decrease in this symptom. One of the two patients $(50 \%)$ with hemoptysis stopped having hemoptysis attacks at the end of the first cycle of treatment. Of the 17 patients with cough, 3 (18\%) showed a reduction in cough (Fig. 2).

Table 2. Comparison of Symptoms, Pulmonary Function TESTS, and HosPitalization Rates ACCORDING TO GENDER

\begin{tabular}{lcccl}
\hline & Patients & Men & Women & \multicolumn{1}{c}{$\mathrm{p}^{\mathrm{a}}$} \\
\hline Cough, $n(\%)$ & $17(63)$ & $10(58.8)$ & $7(41.2)$ & 0.952 \\
Sputum, $n(\%)$ & $22(81.5)$ & $13(59.1)$ & $9(40.9)$ & 0.970 \\
Dyspnea, $n(\%)$ & $19(70.4)$ & $14(73.7)$ & $5(26.3)$ & $\mathbf{0 . 0 1 9} *$ \\
Others, $n(\%)$ & $4(14.8)$ & $1(33.3)$ & $3(66.7)$ & 0.295 \\
FVC \% & $54 \pm 20$ & $50 \pm 22$ & $59 \pm 17$ & 0.287 \\
FEV1 \% & $40 \pm 21$ & $34 \pm 20$ & $49 \pm 18$ & 0.055 \\
Hospitalization & $1.24 \pm 1.36$ & $1.5 \pm 1.6$ & $0.9 \pm 0.9$ & 0.345 \\
$\quad$ rates & & & & \\
$\quad$ frequency & & & & \\
$\quad$ per year) & & & &
\end{tabular}

${ }^{\mathrm{a}}$ Between men and women.

${ }^{\mathrm{b}}$ Two patients: hemoptysis, two patients: wheezing. $* p<0.05$.
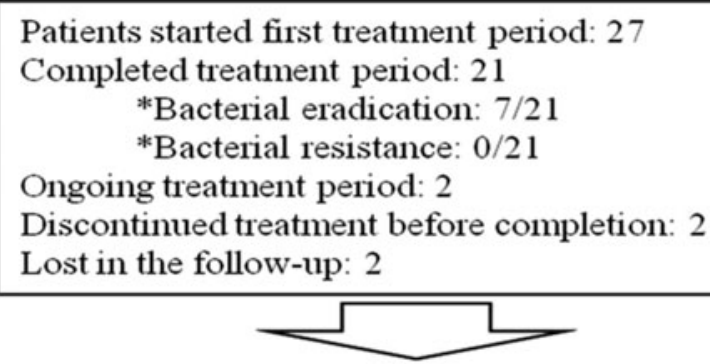

Patients started second treatment period: 14

Completed treatment period: 7

*Bacterial eradication: $2 / 7$

*Bacterial resistance: 1/7

Ongoing treatment period : 7

Discontinued treatment before completion: 0

Lost in the follow-up: 0

\begin{tabular}{l}
$\begin{array}{l}\text { Patients started third treatment period: } 4 \\
\text { Completed treatment period: } 3 \\
\text { *Bacterial eradication: } 0 / 3 \\
\text { *Bacterial resistance: } 0 / 3 \\
\text { Ongoing treatment period: } 1 \\
\text { Discontinued treatment before completion: } 0 \\
\text { Lost in the follow-up: } 0\end{array}$ \\
$\begin{array}{l}\text { Patients started fourth treatment period: } 3 \\
\text { Completed treatment period: } 1 \\
\text { *Bacterial eradication: } 1 / 1 \\
\text { *Bacterial resistance: } 0 / 1 \\
\text { Ongoing treatment period: } 2 \\
\text { Discontinued treatment before completion: } 0 \\
\text { Lost in the follow-up: } 0\end{array}$ \\
\hline
\end{tabular}

FIG. 1. Treatment cycles and eradication ratio in patients receiving inhaled tobramycin are shown in flow chart (ongoing treatment period refers to "the patient whose treatment period is not yet completed").

The frequency of hospitalizations before treatment was $1.24 \pm 1.36$ per year, which decreased to $0.52 \pm 0.91$ per year after treatment, with a significant difference $(p=0.019)$.

FEV1 was $<80 \%$ in the PFT results of all patients before treatment. No statistically significant difference was observed between the PFT values of the patients who completed at least one period before and after treatment. FEV1 was $40 \% \pm 21 \% 1$ month before treatment and $44 \pm 19$ 1 month after the last period was completed; there was no statistical difference $(p=0.97)$. Similarly, FVC was $54 \pm 20$ before treatment and $55 \pm 19$ after treatment; there was no statistical difference $(p=0.29)$. Also, there was no statistical difference according to CRP and white blood cell (WBC) between pre- and post-treatment (Table 3). Patients with eradication of PA were compared with those without eradication in terms of gender, smoking history, and PFTs. All these comparisons were done in patients who had completed at least one treatment period (Table 4). The most common 


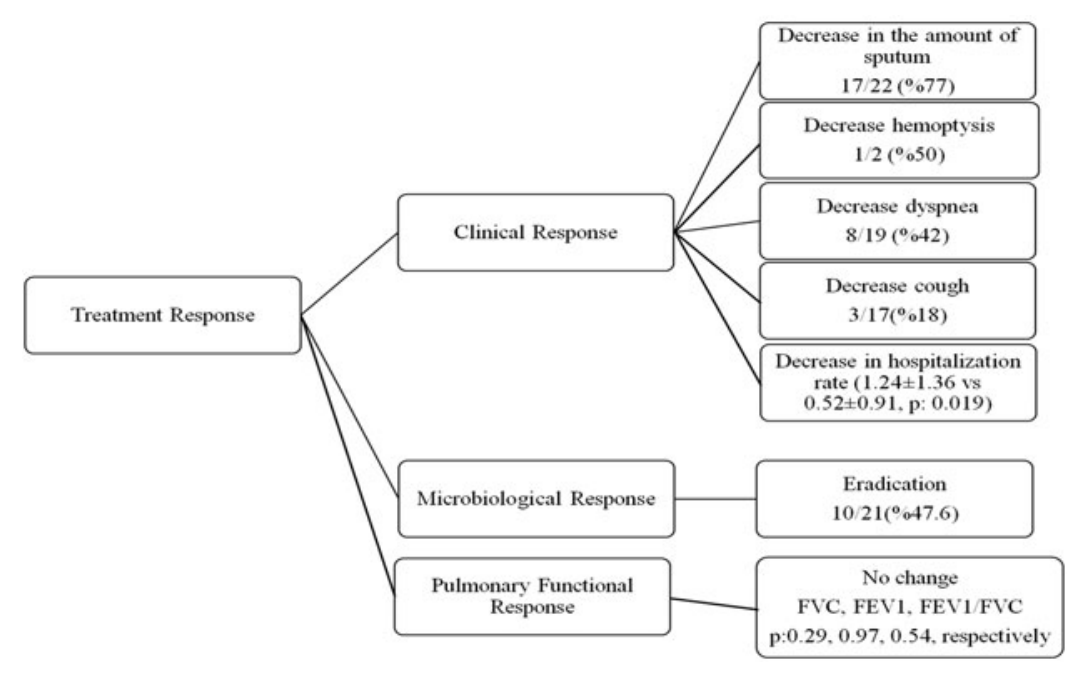

FIG. 2. Clinical, functional, and microbiological evaluation of treatment response in patients receiving tobramycin treatment.

side effect was an increase in dyspnea after nebulization $(n=5)$. Two patients had discontinued the treatment due to this side effect. In three patients, dyspnea did not require cessation of treatment. Two of these patients used the drug once-daily and described clinical relief with reduced sputum purulence. Only two patients did not continue the first 6 months treatment period without any reason. Two patients discontinued their treatment due to side effects. Apart from that, there was no problem regarding treatment compliance in other patients.

\section{Discussion}

In this study, we observed that tobramycin treatment resulted in significant decrease in hospitalization rates in patients with symptomatic NCFB with PA colonization. We also believe this treatment may improve symptoms in these patients.

There are a limited number of studies demonstrating tobramycin usage in patients with NCFB. In a phase II multicenter double-blind randomized placebo-controlled trial conducted by Barker et al. in 2000, $300 \mathrm{mg}$ inhaled tobramycin was used twice daily through a jet nebulizer for 4 weeks. The authors evaluated the quantitative changes in sputum PA density from baseline to the end of therapy. They noted a significant reduction in bacterial load after tobramycin treatment compared with placebo, which correlated with clinical improvement. ${ }^{(8)}$ In another study on 41 patients conducted by Scheinberg and Shore, significant im-

Table 3. Comparison of Pre- and Post-Treatment Pulmonary Function Tests, Leukocyte, and C-Reactive Protein Results

\begin{tabular}{lccc}
\hline & Pre-treatment & Post-treatment & $\mathrm{p}$ \\
\hline FEV1 \% & $40 \pm 21$ & $44 \pm 19$ & 0.970 \\
FVC \% & $54 \pm 20$ & $55 \pm 19$ & 0.290 \\
FEV1/FVC & $59 \pm 14$ & $61 \pm 14$ & 0.545 \\
Leukocyte & $8.38 \pm 4.35$ & $6.97 \pm 5.61$ & 0.341 \\
CRP & $15.13 \pm 17.3$ & $29.34 \pm 34.3$ & 0.289 \\
\hline
\end{tabular}

CRP, C-reactive protein. provements in the quality of life and symptom severity score as measured by St. George's Respiratory Questionnaire (SGRQ) compared with those at baseline were reported after 12 weeks. ${ }^{(9)}$ In our study similar to the literature, according to patients' reports after each cycle, the most common clinical response was a reduction in sputum purulence and quantity, followed by a reduction in dyspnea and cough symptoms.

Orriols et al. conducted a study to investigate the efficacy of tobramycin inhalation therapy in patients with NCFB. After the isolation of $P$. aeruginosa and a 14-day intravenous treatment with ceftazidime and tobramycin, the patients received $300 \mathrm{mg}$ nebulized tobramycin twice daily or placebo for 3 months and were followed up for 12 months thereafter. The number of exacerbations $(p=0.044)$, hospital admissions $(p=0.037)$, and days of hospitalization $(p=0.034)$ were lower in the tobramycin group than in the placebo group in their study. ${ }^{(6)}$ In the randomized placebocontrolled study conducted by Drobnic et al., the number of exacerbations per patient who required admission was significantly lower in the tobramycin group than in the placebo group. Moreover, the number of days of hospital admission per patient was significantly lower in the tobramycin group. ${ }^{(10)}$ Similarly, in our study, there was a significant decrease in the number of hospitalizations with tobramycin treatment. In contrast to the abovementioned studies, we evaluated the frequency of hospitalization retrospectively

Table 4. Comparison of Patients With and Without ERAdication In Terms of Gender, SMoking History, and Pulmonary

Function Tests in Patients Who Have Completed at Least One Treatment Period

\begin{tabular}{lccc}
\hline & $\begin{array}{c}\text { Eradication } \\
(\mathrm{n}=10)\end{array}$ & $\begin{array}{c}\text { eradication } \\
(\mathrm{n}=11)\end{array}$ & $\mathrm{p}$ \\
\hline FEV1 \% & $45 \pm 15$ & $38 \pm 24$ & 0.384 \\
FEV1/FVC & $58 \pm 15$ & $60 \pm 13$ & 0.727 \\
$\begin{array}{l}\text { Smoking history } \\
\quad \text { (smoker/nonsmoker) }\end{array}$ & $2 / 8$ & $4 / 7$ & 0.407 \\
Gender (women/men) & $6 / 4$ & $4 / 7$ & 0.278
\end{tabular}


based on the pretreatment and post-treatment data of the patients, and we found a significant change based on these data.

Bacterial eradication is another parameter that can be used to evaluate the efficacy of tobramycin treatment. Barker et al. reported $35 \%$ bacterial eradication in the tobramycin group, but there was no eradication in the placebo group. Bacterial eradication was evaluated at the end of 6 weeks (after administered twice daily for 4 weeks, followed by 2 weeks off drug) in their study. ${ }^{\left({ }^{8}\right)}$ In another study conducted by Scheinberg and Shore, eradication was achieved in $22.2 \%$ of the patients. They applied three cycles in their treatment protocol. Each treatment cycle consisted of twice-daily dosing for 2 weeks, followed by 2 weeks off treatment, and bacterial eradication was evaluated after three cycles (week 12). ${ }^{(9)}$ In our study, 33\% of the patients had negative sputum culture after the first period (week 24). When positivity was detected after the first period, treatment was continued, and an overall $46.7 \%$ bacterial negativity was achieved. There was one patient receiving four periods and the treatment duration was 96 weeks. In these studies, bacterial eradication was evaluated at different time intervals. We believe that there is a need for studies investigating how long after initiating antibiotic use bacterial eradication can be achieved.

In the studies of Couch and Barker, the efficacy of treatment on PFT could not be demonstrated. ${ }^{(8,11)}$ The mean change in FEV1 and FVC from enrollment to the end of each study period was not significantly different in the study reported by Drobnic et al. ${ }^{(10)}$ Similar to these studies in non$\mathrm{CF}$ patients, we did not find any improvement in the lung function tests.

Nebulized tobramycin sulfate is generally administered for 28 days in two daily doses of $300 \mathrm{mg}$ each, followed by 28 days without tobramycin therapy to reduce the risk of side effects and antibiotic resistance. This regimen was originally tested in patients with $\mathrm{CF} .^{(12)}$ In our clinic, tobramycin treatment is used in the same manner as the abovementioned protocol. This is because, to the best of our knowledge, there is no standardized protocol in the literature for the treatment of tobramycin inhalation in NCFB.

In patients with bronchiectasis, the most important risk factors for colonization with potential pathogenic microorganisms are varicose type bronchiectasis, FEV $1<80 \%$, and diagnosis before the age of 14 years. ${ }^{(4)}$ Since our study was retrospective, the diagnosis time of all patients was not recorded. However, the FEV1 values of all patients were $<80 \%$ and varicose bronchiectasis was observed in $85 \%$ of the patients. Moreover, bronchiectasis was bilateral in the majority of our patients.

Previous studies have used a variety of definitions for chronic colonization in patients with bronchiectasis. These definitions have included at least three isolates of an organism over a period of at least 3 months, and at least two isolates 3 months apart over 1 year or three or more consecutive cultures separated by at least 1 month over a period of 6 months. ${ }^{(13)}$ In our clinic, bacterial growth detected in three consecutive sputum samples at least 1 month apart in the past 6 months was accepted as colonization.

Inhaled tobramycin is generally well tolerated. Respiratory side effects have been reported more frequently in

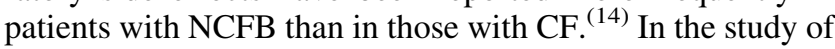
Barker et al., the side effects were higher in the group re- ceiving tobramycin treatment than in the placebo group. Cough, dyspnea, or wheezing due to nebulization occurred in $16 \%$ of patients. ${ }^{(8)}$ In the study of Scheinberg and Shore, cough $(26.8 \%)$, dyspnea $(17.1 \%)$, and wheezing $(17.1 \%)$ have been reported. In total, $22 \%$ of the patients withdrew from the treatment because of side effects. ${ }^{(9)}$

In the study reported by Drobnic et al., 3 of 30 patients (10\%) left the study due to bronchospasm. Tobramycin aerosol therapy was generally well tolerated by the 20 patients who completed the study, and only 1 patient had shortness of breath and wheezing that did not require discontinuation of treatment. ${ }^{(10)}$ The higher incidence of side effects in patients with NCFB than in patients with CF was associated with higher mean age and smoking history. ${ }^{(14)}$ In fact, the mean age of our patients was 50 years and one of three patients had a history of smoking. History of occupational and environmental, particularly biomass, exposure was seen among our patients. The most common side effect was dyspnea $(18.5 \%)$, similar to that reported in the literature. Only two patients discontinued the therapy due to side effects, whereas the other three patients continued the therapy despite the side effects. The therapy was well tolerated in $81.5 \%$ of our patients. The longest duration of treatment was 96 weeks, and there was no renal toxicity or ototoxicity in this patient.

The limitations of our study include lack of the control group, retrospective evaluation, and subjective evaluation of treatment response. No validity scale or quality of life questionnaire was used to evaluate the clinical response. Furthermore, there was no established protocol on the total treatment time and the number of cycles. We believe that our study is important as it is the first to present real life data evaluating long-term antibiotic use and longer follow-up.

As a conclusion, we found that inhaled tobramycin reduces the hospitalization rates. Our results according to declaration of patients showed that symptoms may reduce with this treatment. Also, the microbiologic data demonstrated eradication of PA in $46.7 \%$ of patients, these results may show that treatment may reduce bacterial colonization. To the best of our knowledge, our study had the longest treatment and follow-up duration for patients in the literature. We believe that tobramycin inhalation therapy can be used for clinical improvement and sputum eradication in patients with NCFB. Moreover, it is well tolerated with rare side effects. However, further studies are necessary to establish treatment standardization and determine the place of tobramycin inhalation therapy in the management of NCFB. We believe that our study findings could be a guide for future studies and encourage the researchers who monitor these patients in clinical practice.

\section{Authors' Contributions}

E.C., E.T., and E.G.U.C. designed the research/study. D.T., B.Z.Y., E.T., and S.G. collected data. E.C., H.C., M.A.O., and E.T. analyzed data. E.T., B.Z.Y., and E.G.U.C. wrote the article. H.C., M.A.O., D.T., and S.G. searched the literature. E.C. and M.A.O. critically reviewed the article.

\section{Author Disclosure Statement}

The authors declare they have no conflicting financial interests. 


\section{Funding Information}

The authors received no funding for this study.

\section{References}

1. Satırer O, Mete Yesil A, Emiralioglu N, Tugcu GD, Yalcın E, Dogru D, Kiper N, and Ozcelik U: A review of the etiology and clinical presentation of non-cystic fibrosis bronchiectasis: A tertiary care experience. Respir Med. 2018;137:35-39.

2. Minov J, Karadzinska-Bislimovska J, Vasilevska K, Stoleski S, and Mijakoski D: Assessment of the non-cystic fibrosis bronchiectasis severity: The FACED score vs the Bronchiectasis Severity Index. Open Respir Med J. 2015;9: 46-51.

3. Fjaellegaard K, Sin MD, Browatzki A, and Ulrik CS: Antibiotic therapy for stable non-CF bronchiectasis in adults-A systematic review. Chron Respir Dis. 2017;14: 174-186.

4. Goeminne P, and Dupont L: Non-cystic fibrosis bronchiectasis: Diagnosis and management in 21st century. Postgrad Med J. 2010;86:493-501.

5. Gao YH, Guan WJ, Zhu YN, Chen RC, and Zhang GJ: Antibiotic-resistant Pseudomonas aeruginosa infection in patients with bronchiectasis: Prevalence, risk factors and prognostic implications. Int J Chron Obstruct Pulmon Dis. 2018;13:237-246.

6. Orriols R, Hernando R, Ferrer A, Terradas S, and Montoro B: Eradication therapy against Pseudomonas aeruginosa in non-cystic fibrosis bronchiectasis. Respiration. 2015;90: 299-305.

7. Paredes Aller S, Quittner AL, Salathe MA, and Schmid A: Assessing effects of inhaled antibiotics in adults with noncystic fibrosis bronchiectasis-Experiences from recent clinical trials. Expert Rev Respir Med. 2018;12:769-782.

8. Barker AF, Couch L, Fiel SB, Gotfried MH, Ilowite J, Meyer KC, O’Donnell A, Sahn SA, Smith LJ, Stewart JO, Abuan T, Tully H, Van Dalfsen J, Wells CD, and Quan J: Tobramycin solution for inhalation reduces sputum Pseudomonas aeruginosa density in bronchiectasis. Am J Respir Crit Care Med. 2000;162:481-485.
9. Scheinberg P, and Shore E: A pilot study of the safety and efficacy of tobramycin solution for inhalation in patients with severe bronchiectasis. Chest. 2005;127:1420-1426.

10. Drobnic ME, Suñé P, Montoro JB, Ferrer A, and Orriols R: Inhaled tobramycin in non-cystic fibrosis patients with bronchiectasis and chronic bronchial infection with Pseudomonas aeruginosa. Ann Pharmacother. 2005;39:39-44.

11. Couch LA: Treatment with tobramycin solution for inhalation in bronchiectasis patients with Pseudomonas aeruginosa. Chest. 2001;120:114-117.

12. Hoppentocht M, Akkerman OW, Hagedoorn P, Alffenaar JW, van der Werf TS, Kerstjens HA, Frijlink HW, and de Boer AH: Tolerability and pharmacokinetic evaluation of inhaled dry powder tobramycin free base in non-cystic fibrosis bronchiectasis patients. PLoS One. 2016;11: e0149768.

13. Borekci S, Halis AN, Aygun G, and Musellim B: Bacterial colonization and associated factors in patients with bronchiectasis. Ann Thorac Med. 2016;11:55-59.

14. Vendrell M, Muñoz G, and de Gracia J: Evidence of inhaled tobramycin in non-cystic fibrosis bronchiectasis. Open Respir Med J. 2015;9:30-36.

Received on April 1, 2020

in final form, November 15, 2020

Reviewed by: Timothy Corcoran Lucy Palmer

Address correspondence to: Elif Tanriverdi, $M D$

Department of Pulmonology

Yedikule Pulmonary Diseases and Thoracic Surgery Education and Research Hospital Belgradkapi Street No. 1 Istanbul 34020

Turkey

E-mail: dr.elif06@gmail.com 